EWA PÓŁTORAK

Uniwersytet Śląski w Katowicach

\title{
Question du feedback en didactique des langues étrangères : quelques réflexions théoriques
}

\author{
The Issue of Feedback in the Context of Foreign \\ Language Teaching: a Few Theoretical Reflections
}

\begin{abstract}
The article aims to take the issue of feedback into the context of teaching/learning foreign languages. In the first place, the source concept of feedback will be presented. Then, the feedback phenomenon will be defined in the context of teaching foreign languages. It will allow to discuss its significance and specific roles that it may play for individual participants in the process of teaching/learning foreign languages. In conclusion, based on criteria important from the educational point of view, examples of classifications of feedback, which may be accompanied by both real and virtual foreign language classroom, will be presented.
\end{abstract}

KEYWORDS: feedback, foreign language teaching/learning, computer-assisted teaching/learning, traditional and electronic types of corrective feedback.

\section{REMARQUES PRELIMINAIRES}

En tenant compte de la complexité et de la multiréférentialité du processus d'enseignement/apprentissage des langues étrangères, le déroulement de ce dernier ainsi que son résultat final restent étroitement soumis à l'influence de nombreux facteurs d'origines différentes. En s'appuyant sur les modèles d'enseignement/apprentissage théoriques (voir entre autres Germain 1993 ; Wilczyńska 2005), lesdits facteurs peuvent être recherchés soit du côté de l'apprenant, soit de celui de l'enseignant, ou bien encore ils peuvent s'inscrire dans le contexte du processus d'enseignement/apprentissage déterminé ou résulter de l'ensemble des objectifs visés à atteindre. 
Quelle que soit leur origine, tous ces éléments ainsi que la nature et l'intensité des relations entre eux influent, dans une mesure bien variée, sur l'efficacité du processus pris en considération. L'un des facteurs influençant directement les effets du processus d'enseignement/apprentissage à chacune de ses étapes constitue le phénomène du feedback. Dans le présent article, ledit phénomène nous intéressera aussi bien dans un contexte traditionnel d'enseignement/apprentissage des langues étrangères que celui supporté par les nouvelles technologies de l'information et de la communication (NTIC). Avant d'entrer en détail dans la problématique envisagée, il nous paraît utile de rappeler la conception originelle du phénomène choisi à discuter.

\section{ORIGINES DU FEEDBACK}

La notion de feedback est entrée dans le langage scientifique grâce à l'avènement de la pensée cybernétique dans les années quarante du XXe siècle. C'est à Norman Wiener, père fondateur de cette nouvelle approche scientifique, et ses collaborateurs que l'on doit une formulation savante des principes de feedback, quoique son concept était connu dans différents domaines scientifiques bien avant la naissance de la pensée cybernétique (voir entre autres Durand [1979] 2010 ; Vallée 2007). D'une manière générale, les premiers cybernéticiens conçoivent le feedback comme un processus ayant pour essence un échange constant des informations réciproques (ou des actions en retour) entre différents éléments d'un système donné et cela afin de contrôler et, éventuellement, réguler le fonctionnement de ce dernier (cf. Couffignal 1963). En effet, dans une optique cybernétique, « tout système possède une entrée des informations (input) ainsi qu'une sortie (output) qui rend continuellement compte de l'impact de ces données sur le fonctionnement de l'ensemble» (Godefroid 2008 : 21). On dit alors que les éléments d'un système sont reliés par une causalité circulaire et on appelle le processus de feedback une boucle rétroactive ou une boucle de rétroaction.

Rétroaction

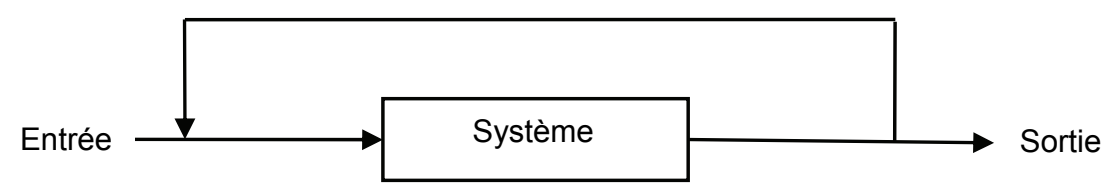

Fig. 1. Schéma d'une boucle de rétroaction (source : Godefroid 2008 : 21) 
De plus, le processus de feedback se déroule toujours en fonction de l'objectif que le système cherche à atteindre (l'accomplissement $\mathrm{d}^{\prime}$ une action par exemple) car c'est de ce but même, inscrit dans le fonctionnement de certains systèmes, que les éléments tirent les informations nécessaires à diriger leurs actions (cf. Wiener et al. 1943). De ce point de vue, le feedback peut être perçu comme un mécanisme permettant à un système donné, qu'il soit naturel ou artificiel, d'assurer l'efficacité de son fonctionnement. Ainsi compris, le concept de feedback a été extrapolé dans divers domaines scientifiques (la psychologie, l'économie, l'informatique, etc.) pour devenir, dès lors, une notion couramment appliquée à toute situation dans laquelle l'objectif visé est une régulation, et donc une modification, d'un comportement donné.

\section{FEEDBACK EN DIDACTIQUE DES LANGUES ETRANGERES}

Traité comme un des éléments essentiels des processus cognitifs de l'homme, le processus d'apprentissage y compris, le feedback a vite gagné une place importante dans les théories d'enseignement/apprentissage. Selon certains chercheurs, toute théorie d'apprentissage envisageant le processus $\mathrm{d}$ 'apprentissage comme un processus d'influences réciproques entre ses acteurs principaux (enseignant/apprenant(s)) se réfère, d'une manière implicite ou explicite, au phénomène du feedback, faute duquel «l'idée même de l'influence réciproque devient par définition impossible » [notre traduction] (cf. Bangert-Drowns et al. 1991, cités par Mory 2004 : 751). De plus, vu le caractère régulateur du feedback, ce dernier peut jouer un rôle important, aussi bien pour l'enseignant que pour l'apprenant, dans le processus d'enseignement/apprentissage de nouveaux savoir, savoir-faire ou de nouvelles compétences en matière donnée (dans notre cas des compétences communicatives langagières et des savoirs culturels en langue cible) et, de par là, influer sur son efficacité.

En didactique des langues étrangères, le feedback peut être alors défini comme un processus permettant à l'enseignant de même qu'à l'apprenant de recevoir différents types d'informations en retour sur la qualité et l'efficacité des activités qu'ils accomplissent tout au long du processus d'enseignement/apprentissage, principalement des activités langagières et communicatives dans le cas de l'apprenant ainsi que des activités didactiques et pédagogiques dans le cas de l'enseignant. Grâce à cela, tous les deux gagnent la possibilité de contrôler lesdites activités et, éventuellement, de les réguler si la réalisation des objectifs visés l'exige. Ainsi compris, le processus de feedback nécessite, d'un côté, l'existence ainsi que la possibilité de choisir 
différents types d'instruments et/ou techniques permettant au destinateur de transmettre des informations en retour ; d'un autre côté, la possibilité, pour le destinataire, d'analyser des informations reçues et, éventuellement, de réajuster ses activités (cf. Galisson, Coste 1976). Aussi bien l'enseignant que l'apprenant peut être, selon le cas, soit destinateur soit destinataire des feedbacks particuliers. Une telle conception du feedback place ce dernier à la source de toute relation didactique, de toute activité, pédagogique, d'une part, et langagière, d'autre part, et ses effets peuvent être analysés aussi bien du côté de l'enseignant que de celui de l'apprenant.

L'enseignant peut recevoir du feedback de ses apprenants par des techniques différentes: soit en demandant directement aux apprenants de donner leurs opinions sur un aspect particulier de l'enseignement, soit en contrôlant leurs progrès (par une observation ou par une évaluation). Quelle que soit la méthode de la collecte des informations, le feedback recueilli peut constituer pour l'enseignant une source d'importantes informations pédagogiques grâce auxquelles il gagne, entre autres, les possibilités :

- de contrôler la qualité et l'efficacité des démarches didactiques entreprises et du processus d'enseignement en général ;

- d'adapter les méthodes d'enseignement proposées aux besoins et aux préférences individuels de ses apprenants ;

- d'individualiser, en cas de difficulté reconnue, le contenu de l'enseignement et/ou les objectifs fixés à courts et/ou à longs termes ;

- d'adapter le rythme d'enseignement aux possibilités des apprenants et/ou à leur niveau de compétences, etc.

Pour l'apprenant, le feedback qu'il obtient de l'enseignant constitue un élément essentiel du processus d'appropriation d'un nouveau savoir, savoirfaire ou d'une nouvelle compétence. Comme le remarque Dakowska (2001 : 37), le feedback est encore plus important dans le processus d'apprentissage d'une langue étrangère, car on ne peut pas progresser dans l'apprentissage si on ne sait pas à quel niveau on se trouve actuellement. Le feedback est alors un élément de prime importance pour assurer le développement des compétences communicatives langagières et, donc, pour assurer l'évolution de l'interlangue.

Par rapport à l'apprenant, le feedback peut être traité, et cela d'une manière très générale, de trois perspectives différentes (cf. Mory 2004). Tout $\mathrm{d}^{\prime}$ abord, le feedback peut être perçu d'une manière purement instructive et, dans ce cas-là, compris comme toute sorte d'information (orale ou écrite, verbale ou non verbale) ou d'activité particulière ayant pour objectif d'informer l'apprenant de la qualité de sa réponse ou, dans un cadre plus large, de ses comportements langagiers et communicatifs. D'un autre côté, et dans un sens plus restreint, le feedback renvoie au processus permettant 
à l'apprenant de comparer le niveau actuel de ses connaissances, compétences, etc., avec celui qu'il cherche à atteindre. Finalement, dans le cas de l'enseignement/apprentissage supporté par NTIC, le feedback désigne toute sorte d'information ou toute une autre forme de réaction du système à l'action de l'apprenant (idem : 745). Quelle que soit la perspective adoptée, le feedback que l'apprenant reçoit de la part de l'enseignant apparaît le plus souvent en littérature didactique au moins sous deux noms: certains chercheurs (voir entre autres Rodet 2000) parlent du processus de régulation, ou d'autorégulation si on situe ce dernier du côté de l'apprenant, d'autres se servent du terme feedback correctif, ce dernier pouvant tout de même porter à l'équivoque. Certes, l'un des objectifs primordiaux du feedback est sa fonction de correction des productions de l'apprenant, celleci étant comprise ici comme une démarche didactique entreprise plus en vue de «porter un jugement le plus objectif possible en fonction d'une norme adaptée (la langue idéale, les réponses attendues à l'exercice, etc.) qu'afin de « relever les écarts en vue de les noter et faire respecter la norme » (Tardieu 2008 : 189). Cette deuxième acception du terme a longtemps régnée dans les conceptions de l'enseignement/apprentissage des langues étrangères en laissant une forte empreinte sur les attitudes envers les productions erronées des apprenants ainsi que les méthodes de leur traitement (voir entre autres Germain 1993). Tout de même, au moins dans les approches actuelles du feedback correctif, ce dernier ne se limite pas à un simple jugement sur l'exactitude de la production de l'apprenant, mais il est aussi censé constituer une aide, pertinente et véritablement profitable, pour l'apprenant dans le processus d'appropriation d'une langue étrangère visée. C'est pourquoi, certains chercheurs trouvent que le feedback, ainsi compris, devrait comporter un élément très important, à savoir un guidage, nommé aussi une remédiation, dont le rôle consiste à " [permettre] à l'apprenant, [d'une part], «de comprendre en quoi sa production est erronée ou déviante » et, de l'autre, «d'essayer de la rectifier» (Gerbault 2010 : 42). Ainsi compris, le feedback requiert le recours à un autre phénomène très important pour le processus d'enseignement/apprentissage des langues étrangères, à savoir l'erreur (voir entre autres Półtorak 2011).

Il faut souligner que le feedback reçu par l'apprenant reste étroitement soumis à des facteurs tels que la nature de l'activité (ou de tâche) langagière mise en œuvre, l'objectif poursuivi, etc., mais aussi les techniques d'enseignement/apprentissage et, ce qui s'ensuit, la conception du processus même d'enseignement/apprentissage, inscrites dans les méthodes et approches existant en didactique des langues étrangères, qu'elles soient traditionnelles ou celles supportées par NTIC (voir entre autres Mory 2004 ; Półtorak 2009, 2011). 


\section{CARACTERE DU FEEDBACK PRESENT EN DIDACTIQUE DES LANGUES CONTEMPORAINE}

$\mathrm{Vu}$ la complexité du processus de feedback, le caractère de ce dernier peut être analysé, dans une perspective didactique, de par le recours aux critères aussi nombreux que diversifiés. Dans le présent article, nous nous sommes limitée à discuter quelques aspects du phénomène choisi trouvés les plus pertinents pour les recherches théoriques visées. Si profondes que soient nos réflexions, elles ne constituent pas une liste exhaustive de tous les paramètres pouvant servir à envisager ou, tout simplement, à classer le feedback en général et celui correctif en particulier.

En prenant en considération les techniques pouvant être utilisées pour procurer du feedback, qu'il aille de l'enseignant à l'apprenant ou dans un sens inverse, nous pouvons distinguer à l'époque contemporaine deux grands types de feedback:

- le feedback traditionnel - propre aux échanges enseignant-apprenant(s) se déroulant d'habitude en classe de langue ou, autrement dit, en présentiel ;

- le feedback électronique - correspondant à toute forme d'échanges enseignant-apprenant(s) par l'intermédiaire des médias électroniques, plus exactement via Internet.

Tout nouveau qu'il soit, le deuxième type de feedback apparaît de plus en plus fréquemment dans le processus d'enseignement/apprentissage des langues étrangères, grâce à une popularité toujours croissante de l'emploi de différents genres de ressources et/ou d'outils multimédias à vocation éducative dans la plupart des contextes didactiques. Alors, il paraît à nos yeux très intéressant, du point de vue didactique, de faire une analyse comparative de ces deux grands types de feedback, ce qui permettra de mettre en lumière leurs principales ressemblances de même que différences et, simultanément, de caractériser globalement le feedback présent en didactique des langues étrangères contemporaine.

Potentiellement, aussi bien le feedback traditionnel que celui électronique peuvent être soumis aux mêmes critères de classification. En conséquence, tous les deux types de feedback peuvent prendre des formes variées selon le mode de communication choisi. Ainsi, les chercheurs distinguent-ils deux formes principales de feedback, qu'il soit traditionnel ou électronique: le feedback verbal, regroupant le feedback oral ainsi que le feedback écrit, et le feedback non-verbal (exigeant, dans le cas du feedback électronique, le recours aux solutions informatiques assez développées, tel que, par exemple, des systèmes de vidéoconférence). En prenant en considération les possibilités offertes par les nouvelles technologies, le feedback peut apparaître 
sous d'autres formes qui activent les canaux auditif et visuel des apprenants : images fixes ou animés, sons, messages audiovisuels, etc. En réalité, ces formes constituent, dans la plupart des cas, une sorte de feedbacks accessoires au feedback électronique principal, celui-ci prenant le plus souvent la forme d'un message écrit (voir entre autres Półtorak 2009).

En passant à un niveau d'analyse plus approfondi, on constate que les résultats de recherches sur le feedback correctif accentuent la manifestation de ce dernier en plusieurs variantes selon le mode de communication choisi par l'enseignant, valables tant pour des contextes didactiques virtuels que ceux réels. Alors, en analysant les messages oraux que les enseignants utilisent en classe de langue pour informer les apprenants de la qualité de leurs productions dans différents types d'activités langagières, Lyster et Ranta (1997 : 37-66) distinguent six types de feedback oral :

- correction explicite d'une forme erronée (explicit correction) : l'enseignant signale directement que l'erreur est apparue et il fournit la forme correcte ;

- reformulation d'une forme erronée (recast) : l'enseignant n'indique pas directement que la forme est incorrecte, mais il ne la laisse pas sans correction non plus : il la corrige implicitement (en la reformulant par exemple) ou il fournit la réponse correcte.

- clarification demandée (clarification request): l'enseignant informe l'apprenant qu'il n'a pas compris son énoncé en recourant aux phrases telles «Pardon?», «Je ne comprends pas. » qui ont pour fonction d'amener l'apprenant à répéter ou à reformuler correctement son énoncé ;

- indice métalinguistique (metalinguistic clue) : encore une fois l'enseignant se sert des questions pour aider l'apprenant à formuler correctement un énoncé mais cette fois-ci les questions sont accompagnées de quelques indices métalinguistiques indiquant, par exemple, que le genre d'un mot donné n'est pas correct ;

- élicitation (elicitation) : technique consistant à faire réagir l'apprenant en lui indiquant qu'un élément donné est incorrect ou inconvenable dans son énoncé à l'aide des phrases telles que "Comment on dit ... en français ? » ou « Le verbe rescuer n'existe pas en français. »;

- répétition d'une forme erronée (repetition): l'enseignant répète la phrase contenant l'erreur en mettant en valeur la présence de cette dernière par une intonation particulière, un ralentissement de la voix, etc.

L'analyse du corpus recueilli a permis à ces chercheurs d'observer que c'est le deuxième type de feedback qui est le plus souvent exploité par les enseignants de langues, sans être pourtant le plus efficace (ibidem). 
Ellis (2009 : 97-107), pour sa part, s'intéresse à la fois au contenu et aux formes que peut prendre le feedback écrit reçu par les apprenants de la part de leur enseignant. Il distingue plusieurs classes de techniques correctives utilisées par ce dernier pour signaler les formes erronées dont celles les plus souvent rencontrées en classe de langue sont les suivantes :

- le feedback direct : propre à la situation où l'enseignant fournit luimême les formes correctes à toutes les formes erronées détectées dans un travail écrit par l'apprenant ;

- le feedback indirect : caractéristique pour la situation où l'enseignant indique que l'erreur est survenue (en la soulignant par exemple) mais il ne la corrige aucunement ;

- le feedback métalinguistique : il apparaît sous forme de commentaires servant à expliquer la nature des erreurs commises ; ces commentaires peuvent être subdivisés en deux catégories : les commentaires basés sur les références aux types d'erreurs commises et exploitant, dans la plupart des cas, les codes des catégories grammaticales correspondantes (le feedback code) et les commentaires faisant référence aux explications métalinguistiques à proprement parler qui, le plus souvent, pour chaque forme erronée indiquent les règles d'usage appropriées (le feedback non-codé);

- le feedback focalisé : technique consistant à ne signaler que les formes erronées d'une catégorie d'erreurs choisie : par exemple, se concentrer, selon les objectifs visés, sur la correction des erreurs linguistiques seulement (toutes ou seulement un type d'erreur choisi) ou uniquement sur les erreurs logiques ;

- le feedback non-focalisé : technique contraire à la précédente et renvoyant à la correction de toutes les erreurs indépendamment de leur type.

Il faut souligner que tous les types de feedback que l'on vient de présenter peuvent se combiner entre eux pour faire émerger de nouvelles formes mixtes telles que le feedback direct focalisé, le feedback codé nonfocalisé, etc.

Parmi d'autres paramètres servant à classifier le feedback correctif, qu'il soit traditionnel ou bien électronique, les chercheurs recourent, entre autres, au critère de temps séparant la réponse de l'apprenant et la réaction de l'enseignant. Ils distinguent alors le feedback immédiat (apparaissant toute suite après la détection d'une forme erronée par l'enseignant) du feedback différé (apparaissant après une période plus ou moins longue) (cf. Mory 2004). Le choix entre les deux types de feedback reste étroitement soumis aux objectifs de l'activité en cours, surtout celles orales : les activités focalisées sur le contenu des informations transmises sont généralement 
suivies d'un feedback différé sauf si les productions des apprenants ne contiennent des erreurs graves, tandis que les activités focalisées sur la forme sont le plus souvent accompagnées d'un feedback immédiat.

Les deux types de feedback correctif envisagés peuvent être aussi divisés selon le public auquel ils sont adressés (ou le(s) destinataire(s)) ou selon le(s) destinateur(s). Dans le premier cas le feedback de l'enseignant peut être adressé soit à un seul apprenant (le feedback individuel), soit à un groupe d'apprenants (le feedback collectif). Dans le deuxième cas, les possibilités sont plus nombreuses: l'apprenant peut recevoir du feedback de son enseignant, d'un autre apprenant, d'un groupe ou bien il peut se corriger soi-même sans intervention d'une tierce personne. Ainsi, parle-t-on du feedback extrinsèque et du feedback intrinsèque (cf. Provencher 1985). Comme pour la catégorie précédente, c'est l'objectif principal de l'activité langagière proposée qui influe sur le choix d'un feedback adéquat. Il faut ajouter encore que, en ce qui concerne le feedback individuel analysé dans un contexte virtuel, certains chercheurs (voir entre autres Sales 1993 [cité par Ollivier, Weiss 2003]) distinguent deux sous-types de ce genre de feedback: le feedback adapté (adapted feedback) et le feedback adaptatif (adaptatif feedback). Le premier type de feedback est basé sur les données introduites par avance dans le programme et qui ne subissent aucune modification au cours de l'exécution du programme. Il est alors stable: chaque apprenant reçoit toujours, pour une même réponse, le même feedback. Par contre, le feedback adaptatif constitue un processus dynamique d'analyse des réponses données par les apprenants. Chaque apprenant reçoit alors un feedback individualisé en fonction de ses réponses antérieures. Selon Ollivier et Weiss (2003), la mise en pratique d'un tel feedback, certes très avantageux pour l'apprenant, présente un vrai défi didactique et technologique : elle exige, d'une part, "une analyse intensive des réponses possibles avant la création du programme ", de l'autre, "une programmation qui permette une analyse de réponse complexe pendant que l'apprenant parcourt les unités ». La seconde contrainte peut être tout de même atténuée, voire écartée, grâce au recours à des outils permettant aux concepteurs des activités interactives de les modifier à tout moment où cela devient nécessaire, tels que par exemple ceux inclus dans les plateformes d'apprentissage (voir entre autres Półtorak 2011).

Outre les paramètres déjà évoqués, les chercheurs s'intéressent au degré de complexité du feedback donné par les enseignants aux apprenants. Ainsi, distinguent-ils cinq niveau de complexité du feedback, qu'il soit pratiqué en présentiel ou par l'intermédiaire de l'ordinateur :

- aucun feedback (no feedback) apparaît dans les cas où l'apprenant est supposé répondre à une question donnée mais il n'obtient aucune information en retour sur la qualité de sa réponse ; 
- simple vérification de la réponse donnée (simple verification feedback ou knowledge of results) : l'apprenant est informé si sa réponse est correcte ou incorrecte ;

- indication de la réponse correcte (correct response knowledge ou knowledge of correct response) : dans ce cas-là, l'apprenant est informé comment devrait être la bonne réponse ;

- feedback élaboré (elaborated feedback) a pour fonction soit de fournir à l'apprenant des informations expliquant pourquoi la réponse donnée est correcte ou incorrecte, soit de lui permettre de revoir certaines parties de l'exercice afin de pouvoir trouver des informations servant à corriger sa réponse ;

- information d'un nouvel essai (try-again feedback) : il informe l'apprenant, dans le cas d'une réponse incorrect, qu'il a à sa disposition un ou plusieurs essais supplémentaires pour pouvoir corriger sa réponse (cf. Mory 2004).

Dans la plupart des cas, toutes les formes de feedback que l'on vient de signaler ne s'excluent pas et le feedback, qu'il soit traditionnel ou électronique, peut apparaître sous différentes formes combinées, ce qui arrive d'ailleurs assez souvent en réalité didactique.

L'analyse que nous avons proposée plus haut met en évidence que les deux grands types de feedback accompagnant le processus d'enseignement/apprentissage des langues étrangères à l'époque actuelle présentent beaucoup de traits communs, voire identiques, en ce qui concerne leur caractère. Bien entendu, ce qui les différencie, c'est principalement leur nature. En effet, on peut dire que, d'une manière générale, ils se distinguent l'un de l'autre de par la nature des relations entre les acteurs principaux du processus didactique, ces dernières étant dans le cas du feedback traditionnel de type réel et, dans le cas du feedback électronique, virtuel. En conséquence, la coexistence de deux types de feedback analysés, tellement pareils mais en même temps tellement distants, peut faire naître des investigations didactiques très intéressantes. Ainsi, la question du feedback en didactique des langues estrangères contemporaine peut-elle se renouveler et acquiert-elle de toutes nouvelles perspectives d'analyse.

\section{EN GUISE DE CONCLUSION}

Les quelques aspects du problème posé que l'on vient d'évoquer montrent l'importance ainsi que le rôle crucial du feedback pour l'efficacité du processus d'enseignement/apprentissage des langues étrangères dans toute sa dimension. La question se pose alors de savoir comment exploiter ou, 
plus exactement, comment améliorer le processus du feedback afin d'augmenter la qualité de l'enseignement/apprentissage d'une langue étrangère donnée. $\mathrm{Vu}$ la complexité du problème pris en considération, la question reste ouverte et les voies de recherches potentielles peuvent prendre des directions bien diversifiées, d'autant plus que la multiplication des techniques d'enseignement/apprentissage, systématiquement enrichies par la mise en place de plus en plus rependues des nouvelles technologies dans le processus d'enseignement/apprentissage des langues étrangères, ouvrent de nouvelles possibilités d'aborder la problématique discutée.

\section{RÉFÉRENCES BIBLIOGRAPHIQUES}

Couffignal, L., 1963. La cybernétique. Paris : PUF.

Dakowska, M., 2001. Psycholingwistyczne podstawy dydaktyki języków obcych. Warszawa: Wydawnictwo Naukowe PWN SA.

Durand, D., [1979] 2010. La systémique. Paris : PUF, coll. « Que sais-je ? ».

Ellis, R., 2009. A typology of written corrective feedback types. In : ELT Journal, 63/2, 97-107.

Galisson, R., Coste, D. (dir.), 1976. Dictionnaire de didactique des langues. Paris: Librairie Hachette.

Gerbault, J., 2010. TIC : panorama des espaces d'interaction et de rétroaction pour l'apprentissage de l'écriture en langue étrangère. In: Revue française de linguistique appliquée, Vol. XV-2, 37-52. Disponible sur : <http://www.cairn.info/revue-francaise-de-linguistiqueappliquee-2010-2.htm>. Dernière consultation : le 15 septembre 2011.

Germain, C., 1993. Evolution de l'enseignement des langues : 5000 ans d'histoire. Paris: Clé International.

Godefroid, J., 2008. Psychologie. Science humaine et science cognitive. Bruxelles : De Boeck Université.

Lyster, R., Ranta, L., 1997. Corrective feedback and learner uptake : Negotiation of form in communicative classrooms. In: Studies in Second Language Acquisition, 19/1, 37-66.

Mory, E.H., 2004. Feadback reasearch revisited. In: Jonanssen D. (eds.): Handbook of research on educational communications and technology. Mahwah: Erlbaum Associated, 745-783.

Ollivier, C., Weiss, G., 2003. Aides à l'apprentissage et retour pédagogique dans les sites d'apprentissage des langues en ligne. In : ASp [En ligne], 41-42/2003. Disponible sur : <http://asp.revues.org/1238>. Dernière consultation: le 15 septembre 2011.

Półtorak, E., 2009. Feedback uzyskiwany drogą elektroniczną. In: Neofilolog, 33, 113-121.

Półtorak, E., 2011. Analyse du feedback dans le processus d'enseignement/apprentissage du français langue étrangère. Thèse du doctorat rédigée sous la direction de Mme Halina Widła, Sosnowiec : Université de Silésie.

Provencher, G., 1985. Les fonctions informatives et les principaux facteurs des feed-back correctifs dans l'apprentissage scolaire. In : Revue des sciences de l'éducation, 11/1, 67-81.

Rodet, J., 2000. La rétroaction, support d'apprentissage ? In : Revue DistanceS, CQFD, 41-74. Disponible sur : <http://cqfd.teluq.uquebec.ca/distances/D4_2_d.pdf>. Dernière consultation : le 15 septembre 2011. 
Rosenbleuth, A., Wiener, N., Bigelow, J., 1943. Behavior, Purpose and Teleology. In: Philosophy of Science, 10, 18-24. Disponible sur : <http://courses.media.mit.edu/2004spring/mas966 /rosenblueth_1943.pdf>. Dernière consultation: le 15 septembre 2011.

Tardieu, C., 2008. La didactique des langues en 4 mots clés : Communication, culture, méthodologie, évaluation. Paris : Ellipses Editions Marketing S.A.

Vallée, R., 2007. Précurseurs de la Cybernétique et de la Systémique. In : La Gouvernance dans les Systèmes. Milan : Polimetrica Publisher, 51-60.

Wilczyńska, W., 2005. Introduction à la didactique du français langue étrangère. Wstęp do dydaktyki języka francuskiego jako obcego. Kraków : FLAIR. 